\title{
Duración del proceso de parición y presentación del feto en el lobo marino común, Otaria flavescens en Punta Negra, Iquique, Chile
}

\author{
Time taken for birthing process and fetus presentation in South American sea lion, \\ Otaria flavescens at Punta Negra, Iquique, Chile \\ Jorge Acevedo $^{1}$ y Anelio Aguayo-Lobo ${ }^{2}$ \\ ${ }^{1}$ Fundación Centro de Estudios del Cuaternario (CEQUA). Avda Bulnes 01890, Punta Arenas, Chile \\ ${ }^{2}$ Departamento Científico, Instituto Antártico Chileno. Plaza Muñoz Gamero 1055, Punta Arenas, Chile \\ jorge.acevedo@cequa.cl
}

\begin{abstract}
Here we document the time involved and the influence of fetus orientation in the birthing process of Otaria flavescens. Our results show that breeching was significantly more frequent than cephalic orientation. The complete birthing process took in average $47.57 \mathrm{~min}$, being the breech orientation significantly longest that the cephalic orientation, particularly from the time of appearance of the amniotic-allantoids sac to
\end{abstract}

placenta in the vulva. Although the adaptive mechanisms involved in the complex process of evolution toward an aquatic life are ongoing, further study of birthing processes are recommended, which could in future provide indicators on such adaptive advantages.

Key words: Cephalic and breech births, reproduction

\section{Introducción}

El proceso evolutivo de adaptación de los mamíferos desde una vida terrestre a una completamente acuática, como es el caso de cetáceos y sirenios, ha requerido de numerosos cambios morfológicos estructurales, fisiológicos, genéticos, ecológicos y reproductivos (Fordyce 2002) durante los últimos 50-54 Ma (Ursing \& Arnason 1998, Domning 2002). Entre estos, la adaptación en la orientación del feto en el nacimiento, desde una presentación típicamente cefálica (mamífero terrestre) a uno típicamente caudal (como los cetáceos), ha otorgado una ventaja para la supervivencia de la cría de los mamíferos marinos actuales, durante el parto bajo el agua (Slijper 1962).

El orden Pinnipedia es un grupo de mamíferos de origen más reciente (11-18 Ma, Berta 2002), encontrándose en una escala intermedia de evolución desde una vida terrestre hacia la acuática, mostrando una combinación de adaptaciones morfológicas y fisiológicas que le permiten la vida acuática, sin embargo la reproducción, y particularmente los nacimientos son aún terrestres.

El proceso de parto en otáridos ocurre anualmente y siempre sobre un sustrato (en playas de arena, roca maciza y bolones), incluyendo playas cubierta por nieve depositada del invierno anterior, como el caso del lobo fino antártico, Arctocephalus gazella (Peters, 1875). A pesar de que muchos aspectos de la biología y fisiología reproductiva son conocidas en la mayoría de las especies de pinnipedios, la duración completa de la parición, comprendida desde la aparición del saco amnioalantoideo hasta la expulsión completa de la placenta al exterior, y la orientación del cachorro en el nacimiento, han sido escasamente documentados; se ha generalizado que la duración del nacimiento en muchas especies de pinnipedios parece ser relativamente corto (Slijper 1956, Bowen 1991). Por su parte, los escasos datos disponibles sobre la orientación del feto en el nacimiento son contradictorios, sugiriendo Harrison (1969) y Bowen (1991) que los nacimientos cefálicos y caudales ocurren en una proporción semejante, mientras que Sweeney (1986) indica que en cautiverio los cachorros normalmente nacen en posición cefálica. Por su parte, en relación a la duración de parto, Harrison (1969) sugiere que la orientación del feto en pinnipedios podría tener alguna incidencia en la extensión del proceso de parto, mostrando Bowen (1991) que los nacimientos de orientación caudal tienden a ser ligeramente más largos que los cefálicos. Sin embargo, Acevedo et al. (2008) muestran para el lobo fino antártico, que los nacimientos de orientación caudal son, en promedio, significativamente más largos (cerca del doble del tiempo) con respecto a aquellos nacimientos de orientación cefálica.

En la zona norte de Chile, la temporada de reproducción y los nacimientos en el lobo marino común, Otaria flavescens (Shaw, 1800), son altamente 
sincronizados, ocurriendo los parimientos mayoritariamente entre enero y febrero (Acevedo et al. 2003). Con respecto al proceso de parición en esta especie, los pocos registros informados en la literatura están referidos en su gran mayoría a la primera fase del proceso llamado parto, es decir, hasta la expulsión completa del feto o nacimiento del cachorro y, que la orientación cefálica sería más común que la caudal (Vaz Ferreira 1975, Vaz Ferreira \& Achaval 1979, Araya et al. 1986).

En este trabajo, se analizó el proceso de parición completo y la orientación del feto, en una colonia de reproducción de lobo marino común en el norte de Chile, como continuación del estudio sobre la reproducción de esta especie, informada previamente por Acevedo et al. (2003).

\section{Material y métodos}

En este trabajo, el proceso de parición completo es definido de acuerdo a Acevedo et al. (2008), como el proceso fisiológico desde la aparición del saco amnioalantoideo hasta la expulsión completa de la placenta, desde el útero a través del canal vaginal al exterior. En este proceso, dichos autores definieron tres fases que son seguidas en este trabajo: a) desde la aparición del saco amnio-alantoideo (ASAA) hasta la expulsión completa del feto, llamado también "parto o nacimiento" (NAC); b) desde el nacimiento hasta la aparición de la placenta en la vulva (APL) y c) desde la aparición de la placenta hasta su expulsión completa al exterior (EPL).

La duración del proceso completo de parición y la presentación del feto al nacimiento, fueron registradas durante dos temporadas consecutivas en la lobera de Punta Negra (2050`S; $70^{\circ} 10^{\prime} \mathrm{W}$ ), localizada a $80 \mathrm{~km}$ al sur de la ciudad de Iquique, norte de Chile (Fig. 1). Para mayores detalles sobre la lobera y eventos de reproducción, ver Acevedo et al. (2003).

Se realizaron observaciones periódicas y continuadas en turnos de dos horas entre censos diarios, usando binoculares 8x40 y un telescopio Tasco ${ }^{\circledR} 21 \mathrm{~EB}$ montado

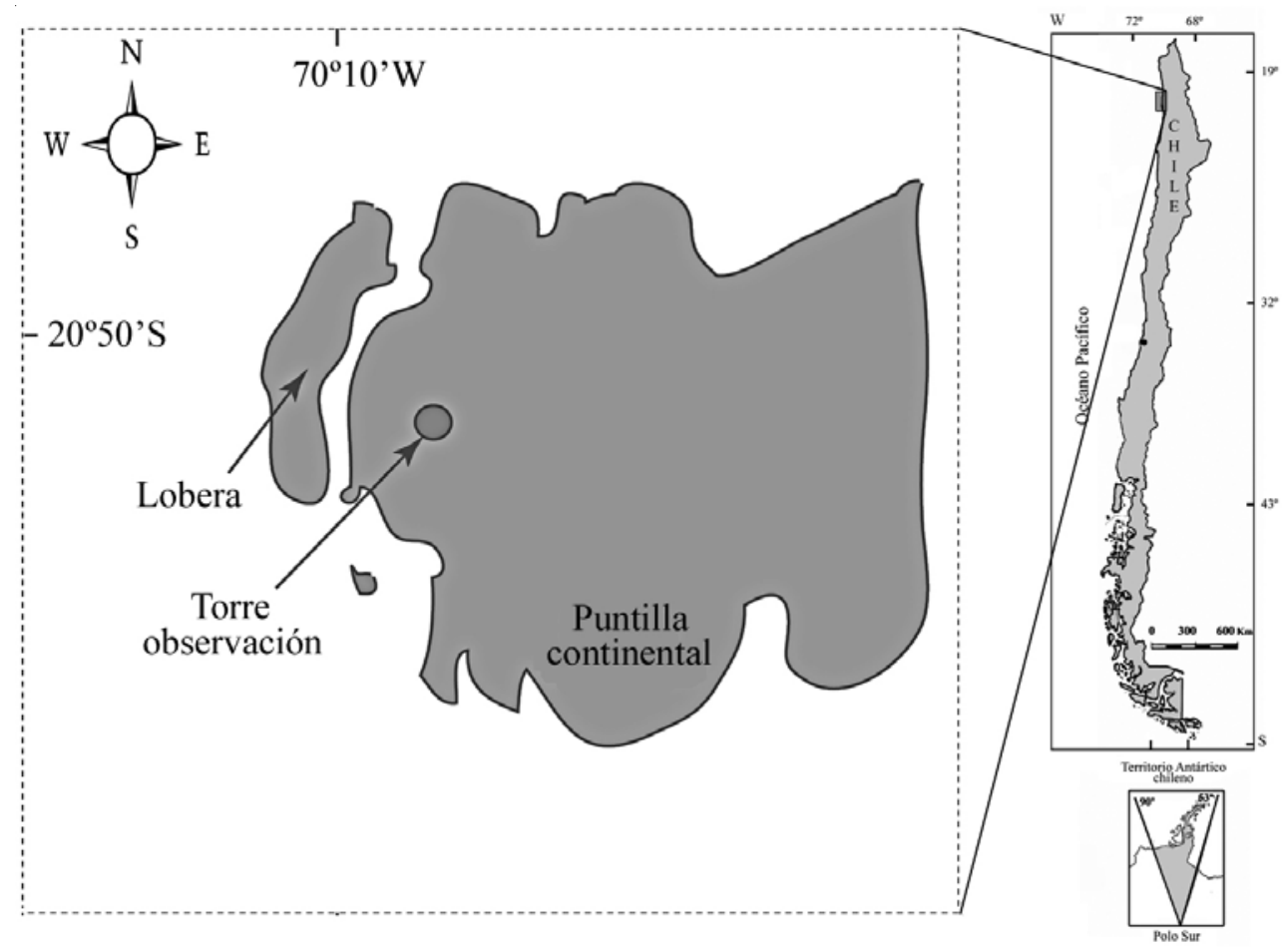

Figura 1

Localización de la lobera de reproducción de Punta Negra (80 km de Iquique), norte de Chile, donde se realizaron las observaciones

Study area showing the breeding islet where the observations were made at Punta Negra (80 km at Iquique), northern of Chile 
sobre un trípode, durante las horas de luz (09:00 a 21:00 $\mathrm{hr}$ ), entre diciembre y marzo de las temporadas 1995/ 1996 y 1996/1997. Conductas de pre-parto en las hembras parturientas (ver Araya et al. 1986, Acevedo 1999) fueron las señales para el seguimiento del proceso de parición completo y se diferenció la orientación del feto al nacimiento en cefálica y caudal.

El proceso de parición completo de las hembras observadas fueron cronometradas y las diferentes fases se determinaron según las anotaciones de campo sobre expulsión de cachorro, así como también la aparición y expulsión de placenta. Adicionalmente, para aquellas hembras donde el proceso de parición no fue cronometrado completamente se consideró sólo la orientación del feto y la duración de la primera fase en el análisis.

Se emplearon análisis estadísticos de probabilidad de proporción $(G)$ para comparar la proporción de nacimientos entre las dos orientaciones (cefálica y caudal), mientras que la prueba no paramétrica de MannWhitney con jerarquía empatada (tied ranks) $(U)$ se usó para comparar la duración del proceso de parición completo y, sus respectivas fases entre ambas orientaciones del feto. Un nivel de significancia de 0,05 (95\%) fue usado en ambas pruebas (Zar 1984).

\section{Resultados y discusión}

\section{Orientación del feto al nacimiento}

Se registró un total de 36 presentaciones fetales, de los cuales el $72 \%$ correspondió a orientaciones caudales. La proporción de nacimientos cefálicos/caudales fue de 1:2,6 nacimientos, siendo esta razón significativamente diferente $(G=7,36$; $d f=1 ; 0,005<P<0,01)$ de la hipótesis de igual proporción de nacimientos (1:1) enunciada por Bowen (1991) para las especies de pinnipedios, y opuestos a lo indicado por Vaz-Ferreira
(1975) y Araya et al. (1986) quienes señalan, en un sentido general, que en $O$. flavescens los nacimientos de presentación cefálica son más comunes que las presentaciones caudales, mostrando que en esta colonia la presentación caudal de los fetos durante el nacimiento sería mucho más frecuente que lo previamente conocido.

Esta mayor proporción de nacimientos caudales por sobre los cefálicos, también ha sido observada en aquellas pocas especies de otáridos donde la cantidad de registros sobre la orientación del feto al nacimiento es mayor a 30 casos. Ejemplo de ellos, son los resultados de Gentry (1970) que informa un 61\% de nacimientos caudales para Eumatopias jubatus (Schreber, 1776); mientras que en Arctocephalus forsteri Lesson, 1828, Crawley \& Wilson (1976) indican una alta ocurrencia de nacimientos caudales (60\%) en isla Snares. Similarmente, Acevedo et al. (2008) informan para A. gazella una mayor y significativa proporción de presentaciones caudales (60\%), en cabo Shirreff, Antártica.

Considerando que $O$. flavescens se encontraría en una escala evolutiva intermedia entre los mamíferos, adaptándose hacia una vida completamente acuática, esta mayor proporción de orientación caudal en los nacimientos, se podría interpretar como un proceso selectivo y necesario para la sobrevivencia de la cría, en la transición desde una vida típicamente terrestre, donde actualmente los nacimientos cefálicos son la norma (Slijper 1962) hacia una vida completamente acuática como los cetáceos, en los cuales los nacimientos caudales son la regla, para evitar el ahogamiento de la cría bajo el agua durante el parto (Slijper 1962, Harrison 1969).

\section{Duración del proceso de parición}

De las 36 presentaciones fetales, se cronometraron 21 pariciones completas (ASAA-EPL) (Tabla 1), la duración de las pariciones completas, considerando la orientación del feto al nacimiento, se presenta en la Tabla 2. La mayor

Tabla 1

Duración (en minutos) del proceso de parición completa y de las respectivas fases sin considerar la presentación del feto de $\boldsymbol{O}$. flavescens en Punta Negra, norte de Chile

Duration (in minutes) of birthing process and each parturition phase, without considering the fetus presentation of $O$. flavescens at Punta Negra, northern of Chile

\begin{tabular}{lcccc}
\hline Eventos & $\mathrm{n}$ & Promedio & $\begin{array}{c}\text { Desviación } \\
\text { estándar }\end{array}$ & Rango \\
\hline Parición completa & 21 & 47,57 & 15,58 & $29-77$ \\
Fase 1 (ASAA-NAC) & 36 & 17,25 & 6,59 & $6-34$ \\
Fase 2 (NAC-APL) & 21 & 14,35 & 7,45 & $4-27$ \\
Fase 3 (APL-EPL) & 21 & 19,12 & 6,20 & $9-35$ \\
\hline
\end{tabular}




\section{Tabla 2}

\section{Duración (en minutos) del proceso de parición y de las respectivas fases, considerando la presentación del feto de $O$. flavescens en Punta Negra, norte de Chile}

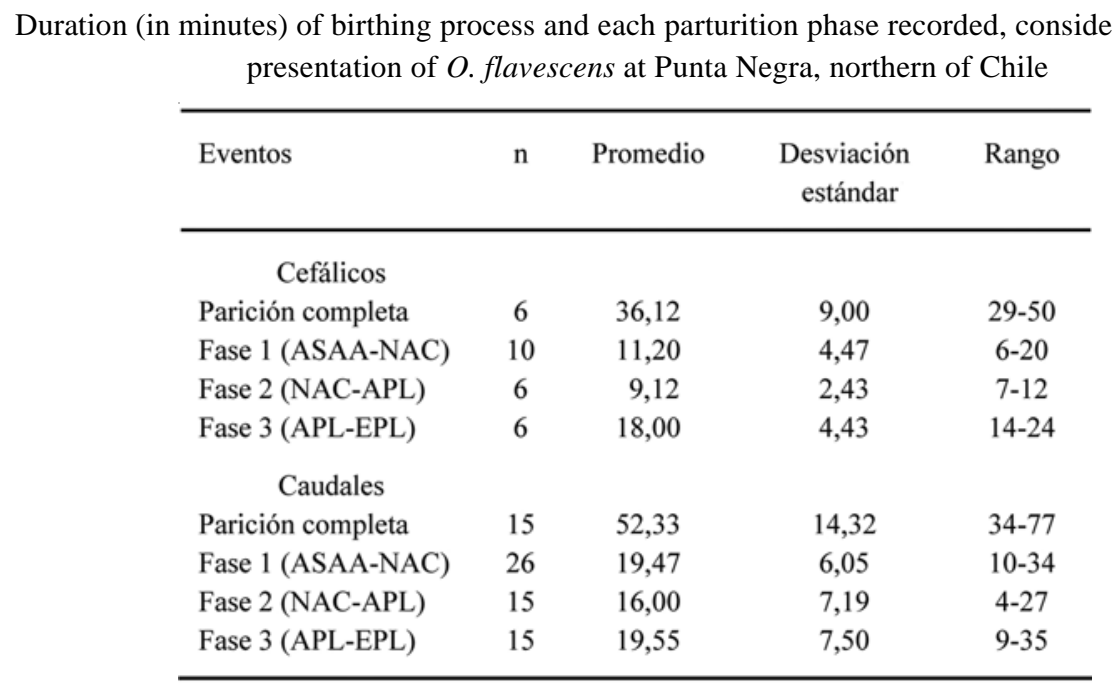

frecuencia de tiempo de las 21 pariciones completas (sin considerar la orientación del feto) resultó entre los 30 y 49 min. Considerando la orientación del feto, el 66,7\% de las pariciones completas de nacimientos cefálicos fueron entre los 29 y $31 \mathrm{~min}$; mientras que la mayor frecuencia $(40,0 \%)$ de las pariciones completas de nacimientos caudales fueron entre los 41 y 49 min.

La duración promedio del proceso de parición completa, sin considerar la orientación del feto al nacimiento, fue de 47,57 min. Además, el tiempo promedio que demora la placenta en ser expulsada completamente después del nacimiento (fases $2+3$ ) fue de 32,48 $\pm 11,05$ min (rango = 20-56 min; $n=21$ ). Al considerar la orientación del feto (Tabla 2), se obtuvieron diferencias significativas $\left(U_{0.05(2), 6,15}=75 ; P=0,02 ; n=\right.$ 21 ) entre la duración promedio de las pariciones de nacimientos cefálicos con respecto a los de nacimientos caudales, indicando que la orientación del feto al nacimiento en esta especie tiene un efecto sobre la duración del proceso de parición completo.

Cuando se analizan separadamente las tres fases del proceso de parición (Tabla 2), el tiempo promedio de nacimiento o parto (fase 1) fue significativamente menor para aquellos de orientación cefálica que para el caso de los nacimientos caudales $\left(U_{0.05(2), 10,26}=220 ; P<0,001\right.$; $n=36$ ), ocurriendo la misma situación al comparar la fase referida desde el nacimiento hasta la aparición de la placenta en la vulva (fase 2$)\left(U_{0.05(2), 6,15}=74 ; 0.02<P<\right.$ $0,05 ; n=21)$. Sin embargo, la última fase mostró una duración promedio similar entre ambas presentaciones fetales. Además, el tiempo promedio en que la placenta es expulsada completamente después del nacimiento (fases $2+3$ ), fue menor aunque no significativamente $\left(U_{0.05(2), 6,15}=67,5 ; 0,05<P<0,10 ; n=21\right)$ para aquellos de nacimientos cefálicos $(\bar{X}=27,12 \pm 6,37 \mathrm{~min} ; n=6)$ con respecto a los nacimientos caudales $(\bar{X}=35,33 \pm$ 11,37 $\mathrm{min} ; n=15$ ).

Antecedentes en $O$. flavescens sobre la duración del proceso de parición completa son escasos y están referidos principalmente al evento de nacimiento (fase 1). Además, debido a eventuales diferencias metodológicas o de criterios empleados por Araya et al. (1986) para esta misma especie y área de estudio, sus resultados se utilizaron sólo para una comparación general. Así, la duración del proceso de parición completa extraída de los resultados de Araya et al. (1986), indican un rango de tiempo de 14 a 57 min. Aunque la duración mínima de nuestros resultados es superior (29 min), los tiempos de parición más frecuentes (30 a 49 min) se encuentran dentro del rango superior señalado por esos autores.

Los resultados del presente estudio sobre la duración del proceso de nacimiento, sin considerar la presentación del feto (6-34 min), se encuentran dentro del rango previamente informado para la especie, entre los 2 y 27 min (Vaz-Ferreira 1975, Vaz-Ferreira \& Achaval 1979, Araya et al. 1986). Los resultados sobre la duración del nacimiento, considerando la presentación del feto, son similares a lo contextualizado por Araya et al. (1986), en 
cuanto a que los nacimientos de presentación cefálica en esta especie serían más breves (4 a $10 \mathrm{~min}$ ) que aquellos de nacimientos de presentación caudal (6 a 27 min). Esta mayor duración en los nacimientos de presentación caudal sería aparentemente la regla en la familia Otariidae, pues observaciones semejantes se han informado para otras especies de otáridos como A. pusillus (Schreber, 1775) (Rand 1955), A. gazella (Acevedo et al. 2008), E. jubatus (Gentry 1970), A. forsteri (Stirling 1971, McNab \& Crawley 1975), Neophoca cinerea (Péron, 1816) y Phocarctos hookeri (Gray, 1844) (Marlow 1975), Zalophus californianus Lesson, 1828 (Odell 1975), recordando que los $n$ son diferentes para las distintas especies señaladas.

La mayor duración en los tiempos de nacimientos caudales por sobre los cefálicos no permiten una fácil interpretación de la ventaja actual que tendría para las especies de pinnipedios; sin embargo, en cetáceos, donde la regla general son los nacimientos caudales, Slijper (1962) informa que los nacimientos pueden tomar entre 30 a 60 min o más. Aunque no se puede descartar la posibilidad de que las diferencias encontradas entre ambas presentaciones fetales, principalmente en la primera fase, pudiera estar condicionada también al estado fisiológico, edad, estrés social u otros factores endógenos de las hembras parturientas, y/o a factores exógenos ambientales, Acevedo et al. (2008) sugirieron dos explicaciones sobre la mayor duración de los nacimientos de presentación caudal para el lobo fino antártico, las cuales también serían válida para el lobo marino común aquí estudiado. La primera, producto de la fricción que se produciría entre la dirección opuesta del pelo del feto y la pared vaginal después de la ruptura del saco amnio-alantoideo durante el tránsito por el canal vaginal; y segundo, a un incremento del diámetro escapular del feto, producto de la posición extendida hacia la cabeza de las aletas pectorales previa ruptura del saco amnio-alantoideo, aumentando la superficie axilar haciendo más dificultoso el tránsito por el canal vaginal hacia el exterior.

A pesar de que la expulsión de la placenta después del nacimiento es considerada en la literatura como un sólo evento, en este trabajo se enmarca en las últimas dos fases del proceso de parición. Sin embargo, nuestros resultados sobre el tiempo transcurrido para la expulsión de la placenta después del nacimiento (fases $2+3)(X=$ 32,5 min; rango = 20 a $56 \mathrm{~min}$ ), son semejantes a Tos rangos informados de 10 (Araya et al. 1986) y $60 \mathrm{~min}$ (Vaz-Ferreira 1975) para esta especie.

Sobre la base de las evidencias presentadas, es posible concluir que la presentación caudal del feto en $O$. flavescens es significativamente mayor que la cefálica, conllevando además a una mayor duración del proceso de parición, particularmente en la fase relacionada con el nacimiento del cachorro (ASAA-NAC). Aunque siguen los mecanismos adaptativos asociados en el complejo proceso evolutivo de una vida acuática, se recomiendan mayores estudios sobre el proceso de nacimiento que podrían proporcionar indicadores de ventajas adaptativas.

\section{Agradecimientos}

Se agradece a la Dirección de la Fundación Centro de Estudios del Cuaternario (CEQUA) e Instituto Antártico Chileno (INACH) por el continuo apoyo de investigación en mamíferos marinos, y por permitirnos dedicar el tiempo necesario para la elaboración de este trabajo. También se agradece a los evaluadores anónimos por la revisión crítica, observaciones y sugerencias otorgadas en este trabajo.

\section{Literatura citada}

Acevedo J. 1999. Temporada de reproducción del lobo marino común, Otaria flavescens (Shaw 1800), en la lobera de Punta Negra (2050`S), Iquique-Chile, durante 1996 y 1997: Crías. Tesis de Biología Marina, Departamento de Ciencias del Mar, Universidad Arturo Prat, Iquique, 150 pp.

Acevedo J, A Aguayo-Lobo \& W Sielfeld. 2003. Eventos reproductivos del león marino común, Otaria flavescens (Shaw, 1800), en el norte de Chile (Pacífico Suroriental). Revista de Biología Marina y Oceanografía 38(2): 69-75.

Acevedo J, A Aguayo-Lobo \& D Torres. 2008. Fetus presentation and time taken for parturition in Arctocephalus gazella at Cape Shirreff, Antarctica. Polar Biology 31(9): 1137-1141 <doi: 10.1007/s00300-008-0446-8>

Araya H, M Arroyo, F Campos \& F Contreras. 1986. Conducta reproductiva del lobo marino común (Otaria flavescens) en Punta Negra, Iquique, Chile. Tesis de Profesor de Estado en Biología y Ciencias, Departamento de Ciencias del Mar, Universidad Arturo Prat, Iquique, 165 pp.

Berta A. 2002. Pinniped evolution. En: Perrin WF, B Wursig \& JGM Thewissen (eds). Encyclopedia of marine mammals, pp. 921-928. Academic Press, San Diego, California.

Bowen W. 1991. Behavioural ecology of pinniped neonates. En: Renouf D (ed). Behaviour of pinniped, pp. 67-127. Chapman \& Hall, London.

Crawley MC \& GJ Wilson. 1976. The natural history and behaviour of the New Zealand fur seal (Arctocephalus forsteri). Tatuara 22(1): 1-29.

Domning DP. 2002. Sirenian evolution. En: Perrin WF, B Wursig \& JGM Thewissen (eds). Encyclopedia of marine 
mammals, pp. 1083-1086. Academic Press, San Diego.

Fordyce RE. 2002. Cetacean evolution. En: Perrin WF, B Wursig \& JGM Thewissen (eds). Encyclopedia of marine mammals, pp. 214-220. Academic Press, San Diego.

Gentry RL. 1970. Social behavior of the Steller sea lion. PhD Thesis, University of California, Santa Cruz, 113 pp.

Harrison RJ. 1969. Reproduction and reproductive organs. En: Andersen HT (ed). The biology of marine mammals, pp. 252-348. Academic Press, New York.

Marlow BJ. 1975. The comparative behaviour of the Australian sea lions Neophoca cinerea and Phocarctos hookeri (Pinnipedia: Otariidae). Mammalia 39: 159-230.

McNab AG \& MC Crawley. 1975. Mother and pup behaviour of the New Zealand fur seal, Arctocephalus forsteri (Lesson). Mauri Ora 3: 77-88.

Odell DK. 1975. Breeding biology of the California sea lion, Zalophus californianus. En: Ronald K \& AW Mansfield (eds). Biology of the seal. Proceedings of a Symposium held in Guelph, 14-17 August 1972. Rapports et ProcèsVerbaux des Rèunions 169: 374-378.

Rand RW. 1955. Reproduction in the female Cape fur seal, Arctocephalus pusillus. Proceedings of the Zoological Society of London 124: 717-740.
Slijper EJ. 1956. Some remarks on gestation and birth in cetacea and other aquatic mammals. Hvalrådets Skrifter 41: $1-62$.

Slijper EJ. 1962. Whales, 475 pp. Hutchinson \& Company, London.

Stirling I. 1971. Studies on the behaviour of the Australian fur seal, Arctocephalus forsteri (Lesson). Australian Journal of Zoology 19: 267-273.

Sweeney J. 1986. Reproduction. En: Murray EF (ed). Zoo and wild animal medicine, pp. 789-790. WB Saunders Company, Philadelphia.

Ursing B \& U Arnason. 1998. Analyses of mitochondrial genomes strongly support a hippopotamus-whale clade. Proceedings of Royal Society of London B 265: 2251-2255.

Vaz-Ferreira R. 1975. Behavior of the Southern sea lion, Otaria flavescens (Shaw) in the Uruguayan Islands. En: Ronald K \& AW Mansfield (eds). Biology of the Seal. Rapports et Procès-Verbaux des Rèunions 169: 219-227.

Vaz-Ferreira R \& F Achaval. 1979. Relación y reconocimiento materno-filial en Otaria flavescens (Shaw) 'Lobo de un pelo’, y reacciones de los machos subadultos ante los cachorros. Acta Zoológica Lilloana 35: 295-302.

Zar H. 1984. Biostatistical analysis, 718 pp. Prentice-Hall, Englewood Cliffs.

Recibido el 05 de agosto de 2009 y aceptado el 28 de diciembre de 2009 